\title{
La psychothérapie déléguée va-t-elle disparaître?
}

\author{
Dr J. de Haller, Médecine générale FMH, Président de la SSMG
}

Dr P. Loeb, Médecine générale, Président AMPP

Dr Ch. Bernath, FMH Psychiatrie et Psychothérapie, Co-Président IGDP

La «Communauté d'intérêt médicale pour la psychothérapie déléguée» (IGDP) a déposé une motion à la Commission de la FMH pour la Formation Post-graduée et Continue (CFPC), visant à la création au niveau fédéral d'un Certificat d'aptitude pour la psychothérapie déléguée. Cette motion prévoyait que les titulaires de ce nouveau Certificat d'aptitude pourraient pratiquer la délégation de la psychothérapie, ainsi que les titulaires du Certificat d'aptitude en Médecine psychosomatique et psychosociale, et que les spécialistes en psychiatrie et psychothérapie ou en psychiatrie et psychothérapie d'enfants et d'adolescent-es.

La CFPC a rejeté cette motion pour plusieurs raisons et avec des objectifs divers. Pour la Société Suisse de Médecine Générale, la psychothérapie déléguée n'est plus que le reliquat d'un temps révolu, qui devrait être abandonné sous réserve de dispositions transitoires acceptables; et aussi parce qu'en tant que prestation nonmédicale, elle n'a plus rien à faire dans un tarif moderne comme le TARMED. La psychothérapie non-médicale, dont la valeur thérapeutique n'en aucunement mise en cause, doit avoir son propre tarif, réglé séparément avec les instances compétentes, comme c'est par exemple le cas pour la physiothérapie. Quant aux psychiatres, ils/elles ont rejeté la motion avant tout en souhaitant poser des exigences notablement plus élevées pour les médecins délégant-es, en l'occurrence une formation complète en psychothérapie.

Actuellement, avant que le TARMED n'entre en vigueur, la loi permet à tout-e médecin de pratiquer la délégation de la psychothérapie. Les assureurs-maladie insistent cependant maintenant déjà sur leur devoir et sur leur droit à poser des exigences de qualité. Ainsi, par exemple, dans le canton de Zürich, l'IGDP et santésuisse ZürichSchaffhouse ont élaboré des critères de garantie tant pour les médecins délégant-es que pour les psychothérapeutes non-médical-es. Cela fait sens et pourrait se négocier selon les besoins, dans les divers cantons.
La somme des motivations citées ci-dessus a fait que c'est pratiquement à l'unanimité que la CFPC a finalement refusé de réglementer à ce sujet, au niveau fédéral, par le biais de la création d'un Certificat d'aptitude. La psychothérapie déléguée devrait être retirée du TARMED, et jusque là elle devrait - dans le sens d'une réglementation transitoire simple et équitable - rester accessible à tou-tes les médecins.

Selon son message du 28.10.2002, le Président de la FMH veut néanmoins laisser en l'état les modalités prévue dans le TARMED 1.1. Cela signifierait qu'avec l'entrée en vigueur du TARMED, seul-es les spécialistes en psychiatrie et psychothérapie ou en psychiatrie et psychothérapie d'enfants et d'adolescent-es pourraient encore pratiquer la délégation. Cela ne correspond d'aucune manière à ce que la CFPC a voulu provoquer par son vote.

Une telle limitation d'une activité médicale, par le biais d'une exigence nouvelle que les praticien-nes concerné-es ne pourraient en aucun cas remplir pendant une période transitoire adéquate, ne correspond pas au droit en vigueur et pourrait être attaquée à tout moment.

Il est clair pour tout le monde que la psychothérapie déléguée, en tant que concept, disparaîtra dès que la psychothérapie non-médicale indépendante sera prise en charge par l'assurance de base. La formulation actuelle du Tarmed signifierait par contre une fin abrupte pour beaucoup de psychothérapies non-médicales en cours. Avant qu'on n'en soit là, nous devons absolument trouver une réglementation transitoire satisfaisante tant pour les médecins que pour les patient-es que pour les psychothérapeutes nonmédicaux/-ales.

La Chambre médicale et la Conférence des Présidents doivent donc reconsidérer cette question de manière urgente et prendre une décision réaliste. 